\title{
Tangence
}

\section{L'instant zéro}

\section{Jean-François Chassay}

Numéro 52, septembre 1996

Tours et détours du romanesque : Minuit aujourd'hui

URI : https://id.erudit.org/iderudit/025916ar

DOI : https://doi.org/10.7202/025916ar

Aller au sommaire du numéro

Éditeur(s)

Tangence

ISSN

0226-9554 (imprimé)

1710-0305 (numérique)

Découvrir la revue

Citer cet article

Chassay, J.-F. (1996). L'instant zéro. Tangence, (52), 53-64.

https://doi.org/10.7202/025916ar

Ce document est protégé par la loi sur le droit d'auteur. L'utilisation des services d'Érudit (y compris la reproduction) est assujettie à sa politique d'utilisation que vous pouvez consulter en ligne.

https://apropos.erudit.org/fr/usagers/politique-dutilisation/
Cet article est diffusé et préservé par Érudit.

Érudit est un consortium interuniversitaire sans but lucratif composé de l’Université de Montréal, l'Université Laval et l'Université du Québec à Montréal. Il a pour mission la promotion et la valorisation de la recherche. https://www.erudit.org/fr/ 


\section{L'instant zéro}

\section{Jean-François Chassay}

Si je me trompe, je suis. Car, certes, celui qui n'est pas ne peut pas se tromper; et j'existe par le fait même que je me trompe.

Saint Augustin

Non. Fiasco. C'était couru. Piètre pédagogue. Peu importe là n'est pas la question.

Daniel Schiff

Depuis toujours, la littérature puise dans l'activité scientifique un certain nombre de modèles, de formes, de métaphores et les écrivains s'inspirent parfois de figures canoniques du monde scientifique pour écrire des textes de fiction (par exemple Edison chez Villiers de l'Isle-Adam, Galilée chez Brecht, Newton chez Stig Dagerman, Copernic, Kepler et Newton dans la trilogie de l'écrivain irlandais John Banville, Einstein récemment chez Alan Lightman, etc.). On a passablement glosé sur les rapprochements possibles entre les transformations de notre perception du monde, provoquées par les discours scientifiques, et la transformation des formes littéraires. Le Nouveau Roman a notamment été abordé dans cette perspective. Pierre Lévy, par exemple, écrit :

On observe dans la littérature une descente vers l'imperceptible comparable à celle que réalise l'informatique. Proust ou Joyce décrivent des événements infimes auparavant négligés par le roman $[\ldots]$ Le nouveau roman a tenté de saisir le continuum de l'expérience humaine sous-jacent aux unités conventionnelles qui fondent le abon sens *. [...] Dans Les corps conducteurs, Claude Simon pousse la discontinuité jusqu'à mener en parallèle près de vingt lignes de description en passant de l'une à l'autre toutes les trois ou quatre phrases. [...] C'est avant tout l'écriture qui se donne à lire, multiple, bourgeonnante, dissolvant les personnages, l'intrigue et les décors, donnant un relief infini au moindre détail ${ }^{1}$.

1 Pierre Lévy, La machine universelle, Paris, La Découverte, 1987, p. 86-87. 
54

Ce type de propos, aujourd'hui assez courant ${ }^{2}$, a peu concerné jusqu'à maintenant la nouvelle génération des écrivains de la maison de Jérôme Lindon. La ligne de Sceaux de Daniel Schiff offre pourtant un portrait assez saisissant d'un physicien. À travers les pensées de celui-ci, c'est également une lecture critique du concept même de science et du langage scientifique que l'auteur propose.

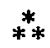

La science moderne est encore relativement jeune. On peut faire remonter sa naissance à la révolution galiléenne, difficilement en-deçà du $X \mathrm{XI}^{\mathrm{e}}$ siècle. Elle a cependant rapidement donné naissance à quelques mythes qui durent et perdurent, malgré les cris d'alarme, souvent ironiques, de nombreux scientifiques, comme Jean-Marc Lévy-Leblond: "En un temps où notre condition, notre vie sont soumises de plein fouet à l'impact de la technoscience, je crois [...] que la littérature peut nous en donner une connaissance "plus complexe et plus juste" que beaucoup d'analyses théoriques [...] Et ceci vaut d'abord pour les scientifiques, dont l'enfermement dans leurs laboratoires ne facilite guère la prise de conscience ${ }^{3}$."

Pourtant, il y a plus de deux siècles déjà que la parole du savant fait autorité, pour le meilleur et pour le pire. Symbole par excellence de la culture pour une frange importante de la bourgeoisie libérale au XviII $^{\mathrm{e}}$ siècle, Isaac Newton ne disparaît pas à l'époque romantique, malgré les fortes réactions au progrès qui se manifestent alors. Il est au contraire plus présent que jamais, mais utilisé à toutes les sauces: le savant devient un démiurge qui a mis au monde des "forces" que les romantiques réutilisent à leur propres fins ${ }^{4}$. Son pouvoir symbolique n'en est que plus grand.

2 Les publications, actes de colloque qui portent sur les rapports entre science et littérature se multiplient depuis une douzaine d'années, surtout aux ÉtatsUnis.

3 Jean-Marc Lévy-Leblond, :Le miroir, la cornue et la pierre de touche *, TLE; 11,1993 , p. 17-18 ["Épistëmocritique et cognition 2n]. Soulignons que JeanMarc Lévy-Leblond est physicien.

4 À ce propos, on pourra lire par exemple, de Georges Gusdorf, L homme romantique, Paris, payot, 1984. Voir aussi, d'Ilya Prigogine et Isabelle Stengers, La nouxelle alliance, Paris, Gallimard, *Folio*, 1979 [1986], notamment le premier chapitre. 
L'image du savant change au cours de la deuxième moitié du $\mathrm{XIX}^{\mathrm{e}}$ siècle, sans que ces qualités de démiurge ne soient remises en question. L'image mythique de Newton sous son arbre, découvrant la gravitation en voyant tomber une pomme, fait place à celle, beaucoup plus prosaique, d'une équipe de chercheurs penchés sur des microscopes et masquant ainsi au commun des mortels ce qu'ils voient. Au $\mathrm{xx}^{\mathrm{e}}$ siècle, tout se passe comme si, voués maintenant à l'imperceptible, les savants devenaient également imperceptibles. Leur fonction et leur pouvoir s'accroissent, leur présence manifeste diminue.

Cette fiction consistant à faire du scientifique l'individu dans l'ombre qui "possède la vérité " a manifestement joué un rôle central dans le mythe et la crainte révérencieuse qui se sont développés à son sujet jusqu'à nos jours. Pourtant, après la théorie de la relativité d'Einstein, le principe d'incertitude d'Heisenberg et le théorème d'incomplétude de Gödel, pour s'en tenir à trois crises importantes ${ }^{5}$, le monde scientifique est beaucoup moins sûr de lui. Reste néanmoins cette impression chez bien des gens (notamment chez de nombreux technocrates des sciences dures) qu'il s'agit du dernier monde où dominent la Raison et une stricte objectivité. Voilà une sphère "utile" de recherches parce qu'on y poursuit des buts précis sur une longue route rectiligne appelée le progrès, grâce à la présence d'êtres désincarnés travaillant dans des laboratoires dont la puissance * se jauge à [leur] capacité de créer des conditions extrêmes: de gigantesques accélérateurs de millions d'électrons-volts, des températures approchant le zéro absolu, des batteries de radiotélescopes couvrant plusieurs kilomètres, [...], des animaleries contenant des milliers de rats ou de cochons d'Inde, de puissants ordinateurs capables de traiter des milliers d'opérations par milli-secondes ${ }^{6}$. "J'ironise à peine. Malgré ses grandes qualités, même un livre comme la Révolution des structures scientifiques de Thomas Kuhn continue de garantir l'autonomie des communautés scientifiques comme si, à l'abri des microbes du discours social, elles pouvaient se protéger, rester pures, en vase clos. Dans ce contexte, la question de la connaissance se vit pendant longtemps réglée par la domination du modèle propre aux sciences ou inspirée par celles-ci.

\footnotetext{
5 Sans compter le rôle des technosciences dans la solution finale nazie et les deux explosions atomiques au Japon.

6 Bruno Latour, La science en action, Paris, La Découverte, 1989, p. 143-144.
} 
Cette vision de la science fut d'ailleurs entérinée, a contrario, par certains littéraires, et non des moindres: Roland Barthes par exemple qui, au milieu des années soixante, opposa la science, modèle hégémonique du savoir érigé en Vérité, et la littérature, terre d'élection du doute, de la polysémie et de l'ambiguité ${ }^{7}$. Les clichés ont la vie dure. Cette lecture manichéenne des Deux cultures - pour reprendre le titre de l'ouvrage de Percy Snow -, une invention de la deuxième moitié du $\mathrm{XIX}^{\mathrm{e}}$ siècle, est une manière d'exclure la science de la culture. Elle devient dans cette optique un phénomène indiscutable, qui dépasse par sa puissance et sa rationalité le commun des mortels et ne mérite pas qu'on s'y arrête. La porosité des frontières entre les discours ne la toucherait pas, la transparence absolue de son langage serait une condition sine qua non de son existence.

C'est bien cette mythologie inébranlable de la science dans le discours occidental que vient joyeusement démolir Daniel Schiff avec son petit roman La ligne de Sceaux publié en 1982 aux éditions de Minuit. Roman de la banalité, de l'ennui (volontairement) étale, il met en scène l'anti-héros scientifique par excellence, le fonctionnaire-chercheur au quotidien. Fidèle aux habitudes éditoriales de la maison de Jérôme Lindon, ce roman ne vise pas une critique du monde des laboratoires à travers les règles de l'illusion référentielle chère à l'esthétique vériste, mais propose plutôt une mise en scène (qui est aussi, dans une certaine mesure, une mise en crise) du langage du chercheur scientifique.

Marc Wolf, quarante-deux ans, physicien, spécialiste des électrons dans les métaux, est un homme d'un inintérêt à peu près complet. À la lecture de certaines réflexions qu'il élabore sur son travail, on l'imagine bien incarner l'auteur laborieux dont parle Lévy-Leblond: "Ce serait encore une étude à entreprendre que d'analyser la pauvreté, le schématisme, la médiocrité de l'écriture scientifique actuelle. La rigide codification formelle qui est de règle aujourd'hui dans les publications professionnelles des chercheurs s'accompagne, on peut le montrer, d'un appauvrissement de la pensée et d'un affaiblissement de l'échange ". La ligne de

7 Roland Barthes, "De la science à la littérature , Le bratsement de la langue, Paris, 1984, p. 13-20. Publié pour la première fois en 1967 dans le Times Literary Supplement.

8 Jean-Marc Lévy-Leblond, - Le miroir, la comue et la pierre de touche ", op. cit., p. 37-38. 
Sceaux, cependant, n'est pas un article scientifique mais bien un roman. Évidence générique qui a son importance, dans la mesure où on peut y voir certaines des possibilités cognitives de la fiction. Si l'essentiel de cet ouvrage de 142 pages est constitué de réflexions sur certaines lois de la chimie ou de la physique et sur le travail scientifique, celles-ci n'apparaissent jamais de manière ordonnée (sauf pour de courts passages où l'entropie semble jugulée) et sont sans cesse traversées par des observations d'une tout autre nature qui n'ont aucune valeur heuristique (du moins directement).

Le lecteur ne voit jamais Marc Wolf travailler, il l'observe en train de penser. Son esprit fonctionne continuellement, dans des conditions souvent difficiles, et se nourrit de tout ce qui l'entoure, y compris d'éléments discursifs parfois fort inconvenants pour un chercheur patenté.

Le roman sape avec efficacité la figure du savant comme autorité tout au long de la narration. Dès les premières pages, il montre le narrateur allongé sur le divan. Fébrile, nerveux, incapable de prendre la parole, Wolf apparaît d'entrée de jeu comme un homme en état d'infériorité, sur la défensive. Son seul pouvoir consiste à se taire, malgré les exortations de Grosjean, son analyste. Et ses pensées oscillent, comme ce sera le cas du début à la fin du texte, entre le sexe et la science ou, si l'on préfère, entre des corps aux contours différents. Il imagine par exemple un homme qui suit une femme:

Moi j'en parle, des mots. Lui la suit. En même temps qu'il monte il regarde. C'est normal. Regarde ses hanches. Ses jambes. Ses fesses. Son cul. Toute la matière, sans exception, est faite d'atomes.

Toute la matière. Des petites boules. Qui gigotent à toute vitesse. Elles sont petites. Minuscules. Microscopiques. Bon donner un chiffre. Tant pis. Un chiffre. On n'y coupe pas. Un millionième de millimètre. Bon ${ }^{9}$.

Tout son univers est chiffré, marqué par le mouvement des corps... et parfois, plus spécifiquement, par le déhanchement de ceux-ci. Ce va-et-vient entre vie privée et vie professionnelle, entre affect et intellect, permet au lecteur d'apprendre peu à peu

9 Daniel Schiff, La ligne de Sceatax, Paris, Minuit, 1982, p. 13. Je me contenterai dorénavant d'indiquer la page entre parenthèses après la citation. 
en quoi consiste son travail mais lui signale également, malgré l'absence à peu près complète de données d'ordre biographique sur Marc Wolf, qu'il s'agit d'un homme banal, obsédé par les femmes (ce qui, en soi, n'est guère original), ayant des fantasmes plutôt quelconques et prévisibles. Le comique du texte vient moins de la volonté du personnage d'être drôle que des associations d'idées qui se forment tout naturellement dans son esprit:

Il semblerait [...] que lorsque nous disons l'électron est passé à la fois par ce trou-ci et par ce trou-là, pour prendre l'exemple des fentes d'Young, nous donnions là, par cet énoncé, non seulement de notre connaissance de la position de l'électron à l'instant en question, mais aussi de la réalité de l'électron à cet instant, de sa réalité intrinsèque, de sa réalité en-soi, indépendante de tout observateur, la description la plus complète possible fin de citation. Elle fait glisser sa culotte. L'idée de la culotte. La culotte en soi laisse tomber. Quel coup pour notre Weltanschauung. (p. 20)

À quoi fait référence ici la Weltanschauung? À la réalité intrinsèque de l'électron ou à la chute de la petite culotte? Est-ce que les "trous " et les "fentes" visent effectivement la réflexion sur l'électron ou est-ce une manière détournée de revenir à la femme? À moins que ce ne soit l'électron qui fasse songer à celle-ci, ou vice-versa...Il y a quelque chose de profondément trivial dans la manière dont Marc Wolf exprime ses idées. Passer des équations de Schrödinger aux jambes d'Yvonne n'apparaît pas très "noble" sans doute. Mais la trivialité est le mode même de fonctionnement de la pensée, celle-ci ne pouvant exister sans mélanges. Les déplacements dans la pensée innovent et on pourrait dire, globalement, que l'intuition heuristique a un caractère analogique. L'esprit est une constellation qui intègre à mesure les éléments qui lui sont nécessaires pour donner éventuellement une direction, un sens à la réflexion. «Le langage est important [...], outre la puissance suggestive de son vocabulaire, parce qu'il est un tissu, un système discursif indépendant du raisonnement purement logique. Un discours quelconque peut être mal cohérent sans être par là même dépourvu de sens ${ }^{10}$." Ainsi, dans le contexte du roman, l'énumération qui suit n'apparaît pas gratuite:

Progrès comprendre la chute des feuilles avancée des connaissances les cristaux liquides les ultra-sons la chute des cheveux. 
Quoi encore. La schizophrénie les accélérateurs les trous noirs les micro-ondes. Connaitre comprendre progrès la ferroélectricité les macromolécules la dérive des continents. Quoi encore. Ah oui les chambres à fils. Les verres de spin. Les nombres premiers les anti-dépresseurs la superfluidité les parfums le langage la mémoire. Ah la mémoire. Comprendre comment ça marche. Les micro-processeurs l'arc-en-ciel. (p. 43)

Ce télescopage narratif qui associe le progrès à la chute des feuilles (qui n'en est pas un), des phénomènes naturels (de la perte des cheveux à la dérive des continents) à des découvertes liées à des problèmes humains (de la compréhension du phénomène de la schizophrénie aux anti-dépresseurs) en passant par des objets utilitaires (les micro-ondes, les micro-processeurs), aboutit tout naturellement à l'image de l'arc-en-ciel, offrant toutes les couleurs du prisme. La mémoire comme répertoire ( Ah la mémoire ") impose cet "arrière-monde" dans l'esprit du scientifique. Immergé dans le cadre social qui est le sien et qui déborde bien sûr largement de ses propres travaux, Wolf ne peut compartimenter complètement son esprit, malgré les équations sur lesquelles il travaille de manière obsessive. "Dans toute société la production du discours est à la fois contrôlée, sélectionnée, organisée et redistribuée par un certain nombre de procédures qui ont pour rôle d'en conjurer les pouvoirs et les dangers, d'en maîtriser l'événement aléatoire, d'en esquiver la lourde, la redoutable matérialité ${ }^{11}$. Or, c'est bien un peu le rôle de ce qu'on nomme littérature de proposer, comme dans La ligne de Sceaux, des rapprochements que la logique sociale, que la logique discursive, n'admettent pas aisément. Pas plus que le discours littéraire, le discours scientifique ne peut s'élaborer de manière immanente et le roman de Schiff le montre parcouru de réflexions hétérogènes. "Ah les conditions d'émergence de la langue" (p. 49), se dit Marc Wolf, dans un passage où, tout à la fois, il tente de définir la couleur des cheveux d'une femme qu'il croise et un paradigme.

Les épiphanies permettent de traverser la pensée de Wolf. Mais surtout, à travers la narration, le lecteur est à même de constater à quel point l'objet scientifique se construit peu à peu à travers le langage dont la neutralité apparaît comme un leurre. Ironiquement, lorsque le narrateur énumère des faits supposément objectifs, il adopte un ton professoral et se met à dialoguer avec

11 Michel Foucault, L'ordre du discours, Paris, Gallimard, 1971, p. 10. 
lui-même, comme si la dimension pédagogique de son propos le réclamait : "On sait que l'uranium deux cent trente-huit est un isotope légèrement instable. [...] Dois-je poursuivre. Oui oui ce n'est pas le moment de laisser tomber. (p. 47) Professeur et élève à la fois, Wolf relance sans cesse son discours, s'interrogeant sur l'utile, le nécessaire et le trivial. Même (et peut-être surtout) dans les passages qui nécessitent le moins d'effets rhétoriques, Wolf sent le besoin d'insister sur l'aspect narratif de son propos. Sans doute est-il "piètre pédagogue "(il l'avoue sans ambages) mais "là n'est pas la question". Si pédagogie il y a, elle repose sur les effets du discours de Wolf qui montre bien l'obscurité et la complexité du langage scientifique, même s'il affirme: "Les nombres voilà où je voulais en venir c'est net c'est rigoureux vous faites la somme soit encore une fois pour sentir." (p. 61)

Grâce à la résistance de nombreux scientifiques,

[l]e discours scientifique semble toujours jouir, comme au siècle dernier le roman réaliste, d'un statut privilégié qui nous retient de le considérer comme une pratique langagière et textuelle à part entière, ayant ses propres règles de fonctionnement, ses propres manières de constituer l'objet dont il parle comme "exprimable" dans des formes d'énoncés qui le caractérisent ${ }^{12}$.

La ligne de Sceaux vise à démontrer la friabilité de ce statut particulier. "En réalité. Quel mot." (p. 93) Le discours scientifique ne jouit pas d'une supériorité intrinsèque et il $\mathrm{y}$ a longtemps que la recherche ne repose plus, pour l'essentiel, sur des "faits objectifs. irréfutables et sur des données empiriques. Que la science soit un langage, qu'elle ne se refuse pas à jouer avec son propre processus de formalisation, est aujourd'hui reconnu au point où il arrive qu'on parle de la valeur *esthétique * d'une équation. "Aesthetics rather than logical is the distinguishing feature of the mathematical mind ${ }^{13}$,. N'est-ce pas ce qu'affirme le narrateur luimême, en parlant narcissiquement des "oscillations de Wolf"?

12 Pierre Ouellet, Voir et satoir. La perception des untvers du discours, Candiac, Balzac, 1992, p. 443.

13 Seymour A. Papert, - The Mathematical Unconscious •, On Aestbetic in Science, Cambridge, M.I.T. Press, 1978, p. 135. Ceci rappelle une scène du roman de Daniele del Giudice, Atlas occidental, qui met face à face cleux personnages: un vieil écrivain américain et un jeune physicien italien. Lorsque le premier demande au second de lui expliquer son travail, celui-ci tente de vulgariser (maladroitement) ses recherches pour être compréhensible. Mais l'écrivain lui dit qu'il ne comprendra pas de toute manière les détails et 
Comment sans cela faire comprendre faire sentir ce désordre des électrons au sein du métal grouillant en tous sens. Quel fouillis. Mais sous-jacent quel ordre quelle beauté, quel calme la courbe jaillie du fond du désordre. Pure. Ligne continue merveilleusement lisse. Fine. Plus fine qu'un cheveu. Faire comprendre si fine justement à cause du désordre sous-jacent des quelque dix à la puissance vingt-trois électrons. (p. 71)

Si les réflexions de Marc Wolf déstabilisent la cohérence attendue du discours scientifique, elles remettent également en question l'autorité du savant et le prestige de la science. Quelle est la secousse sismique qui met en branle les méditations de Wolf? Les raisons de son retour au point zéro, la tabula rasa qui le justifie de retourner au fondement de ses connaissances scientifiques et de se lancer dans une surenchère de chiffres?

À la suite de critiques d'un de ses confrères, il recommence les calculs qui l'ont conduit à publier un certain article, important dans sa carrière. Il doit constater, à son grand dam, qu'une erreur s'y est glissée, remettant totalement en question ses hypothèses. Non seulement le lecteur constate que le scientifique a fait une erreur grave, que son article est obsolète mais, pire encore, la chose n'a aucune importance! Wolf le dit lui-même naïvement, en parlant de son départ du bureau: "L'erreur de signe, finalement, tout le monde était prêt à l'oublier. Mais c'était mes questions. Toujours les mêmes. Toujours les mêmes mauvaises. Genre à quoi bon et ainsi de suite. À la fin ils en ont eu assez ils m'ont changé de bâtiment je les comprends. Je me mets à leur place. J'étais devenu impossible. (p. 119-120) Ce n'est pas la piètre qualité de son travail mais bien plutôt la piètre qualité de son moral qui lui fait perdre son emploi.

La lecture de La ligne de Sceaux provoque des effets paradoxaux. D'une part, le roman contribue à détruire une vision romantique du scientifique, symétrique à celle de l'écrivain: au poète assis sur son rocher face à la mer, les cheveux soulevés par le vent, qui écrirait soudain cinquante sonnets brûlants d'amour grâce à l'intervention de l'inspiration divine, correspondrait le

préfère se faire raconter les choses comme s'il était lui-même physicien. Le jeune Italien se met donc à raconter, spontanément, ses recherches en cours. Ce langage étonnant, merveilleurx, totalement étranger à ce qu'il connaît, apparaît à l'Américain comme de la pure poésie. Daniele del Giudice, Atlas occidental, Paris, Seuil, 1987. 
scientifique froid, technicien, méticuleux, rivé à son écran cathodique qui chercherait (et trouverait, cela va de soi) la Vérité, son langage reposant sur des critères d'exactitude absolue. Ces images caricaturales sont pourtant encore largement véhiculées. L'écrivain, pur de toutes scories sociales, écrivant sur la base de l'inspiration, dans une naturelle immanence ${ }^{14}$, alors que le scientifique est un être dont les propos sont *sûrs" et qui guide tout aussi naturellement le monde vers le progrès.

D'autre part, en rendant compte des avancées et des reculs de la pensée de Wolf, de ses hésitations et de ses brèves illuminations, Daniel Schiff rend beaucoup plus crédible cette figure du scientifique, qui est en fait la représentation d'une pensée en action. La pensée, "l'invention intellectuelle", pour reprendre le titre du livre de Judith Schlanger, fonctionne à la fois grâce à l'intuition, à l'imagination, à l'expérience, à la connaissance et au désir, ceci étant indissociable du contexte discursif dans lequel baigne l'individu. Aucune dichotomie ne peut véritablement prévaloir en ce sens entre le travail du physicien et celui du romancier par exemple.

Certains passages du roman de Daniel Schiff peuvent paraître un peu austères au lecteur qui s'intéresse peu aux angströms et qui serait bien en peine de dire si Fermi était physicien, chimiste ou mathématicien. Mais les réflexions scientifiques s'intègrent toujours à une fiction, à un langage qui s'inventent au fil du texte, dans la mesure où les affres de la vie pour Wolf, sa psychologie, ses fantasmes, viennent sans cesse interférer dans la logique de l'argumentation scientifique. Le texte réinvente, par le fait même, les "faits" scientifiques qui sont sans cesse déplacés et réinterprétés selon une autre logique, propre à la subjectivité d'un homme paumé qui remet jusqu'à un certain point sa vie en question. "Il est [...] bien difficile de dire le vrai sur le faux. Il est même difficile de dire le vrai sur le non vrai, quand l'histoire n'a pas encore donné au vrai, donc au non vrai, son statut de vérité ${ }^{15}$. "À l'ère postmoderne, il existe un risque réel de tomber dans le relativisme absolu, qui est peut-être bien le pire danger de notre

14 C'étaient exactement les propos tenus encore tout récemment par Pierre Vadeboncour qui répliquait dans Le Devotr à Robert Lévesque, ce dernier ayant osé parler de la dimension sociale du travail de l'écrivain.

15 Stella Baruk, L'âge du capitaine. De l'erreur en mathématique, Paris, Seuil, 1985 , p. 40. 
époque. Mais dire que le discours scientifique n'est pas "absolu", qu'il ne peut signifier la Vérité et la Raison en soi vise d'abord, tout au contraire du relativisme absolu, à exercer un esprit critique constant, en prenant notamment en considération le fait que la science est aussi langage, et par le fait même fiction. Ceci se situe d'ailleurs dans un travail de relecture de l'histoire des sciences, à l'aune des bouleversements épistémologiques contemporains:

Nous assistons, au cours de ces dernières années, à la production d'une possibilité nouvelle d'histoire. Aux yeux de certains, l'usage des mathématiques comme instrument de fiction pourrait bien constituer le nouvel avenir, qui reléguerait notre passé et notre présent "galiléen " au statut de période transitoire dont la parenthèse est près de se refermer.

Cette mise en perspective nouvelle est liée au développement des techniques informatiques. En effet, la puissance de l'ordinateur en tant qu'instrument de simulation fait surgir, parmi les scientifiques, ce qu'on pourrait appeler de nouveaux sophistes., des chercheurs dont l'engagement ne se réfère plus à une vérité qui ferait taire les fictions, mais à la possibilité, quel que soit le phénomène, de construire la fiction mathématique qui le reproduit ${ }^{16}$.

On pourrait avancer qu'aujourd'hui *Vérité, réalité et démarche s'engagent mutuellement dans une opération qui crée des récits là où nous comprenions par jugement ${ }^{17}$. et on considère de plus en plus certains acquis de la science d'abord comme des formes de récit, si on se place du point de vue du modèle théorico-expérimental " dur * la biologie darwinienne par exemple ${ }^{18}$.

Le roman de Daniel Schiff ne discute pas explicitement des questions soulevées ici par mes propos. Il ne s'agit pas d'une thèse et, rappelons-le, Marc Wolf affirme explicitement être "piètre pédagogue". Mais, tout aussi explicitement, il ajoute : * peu importe là n'est pas la question. En effet, le roman se contente - mais ce n'est pas rien - de montrer une pensée en train

16 Isabelle Stengers, L'invention des sciences modernes, Paris, La Découverte, "Armillaire., 1993, p. 153.

17 Ibid., p. 161.

18 Sur cette -mise en récit " et son développement, on peut lire bien sûr les excellents livres de Stephen Jay Gould. Voir aussi l'article de Paul Dumouchel, * Le rôle de la fiction en biologie évolutionniste •, TLE, «Épistémocritique et cognition 2., 11, septembre 1993, p. 73-86. 
64

de s'élaborer et, à travers son langage, de montrer comment la pensée s'élabore. La réflexion scientifique, la plus "rationelle" croit-on, la plus à même d'échapper aux risques de la fiction, c'est-à-dire de la réinvention de son propre univers discursif, en apparaît au contraire comme le modèle. Lu dans cette perspective, le texte littéraire apparaît non pas comme un objet pour une discipline académique au sens traditionnel ou entendu, mais plutôt comme un point de départ pour un travail de contextualisation et d'invention, un mode de savoir particulier sur le monde. 